\title{
A hybrid equalizer/Rake receiver for the Wideband CDMA Downlink in large delay spread channels
}

\author{
C. Buchacher ${ }^{1}$, M. Zimmermann ${ }^{1}$, S. Paul ${ }^{2}$, and M. Huemer ${ }^{3}$ \\ ${ }^{1}$ Infineon Technologies, Am Campeon 1-12, 85579 Neubiberg, Germany \\ ${ }^{2}$ Institut für Theoretische Elektrotechnik und Mikroelektronik, Univ. of Bremen, Otto-Hahn Allee, 28359 Bremen, Germany \\ ${ }^{3}$ Embedded Systems and Signal Processing Group, University of Klagenfurt, Lakeside B02b, 9020 Klagenfurt, Austria
}

\begin{abstract}
Signals transmitted over a multi-path propagation channel exhibit Inter-Path Interference (IPI) and fading. The receiver has to employ measures to mitigate these effects or it will incur severe performance degradation. A classic approach in Code Division Multiple Access (CDMA) communications is the Rake receiver. Alternatively, a Linear Minimum Mean Square Error (LMMSE) equalizer can further improve performance. This paper compares performance and computational complexity of these two algorithms. A hybrid solution will be introduced in order to decrease computational complexity while retaining most of the interference suppression capabilities of the LMMSE equalizer.
\end{abstract}

\section{Introduction}

Spread-spectrum signals inherently exhibit frequency diversity. Due to the increased bandwidth, a spectral null is less likely to affect the entire signal spectrum. For the same reason, however, the radio channel is likely dispersive. In order to exploit frequency diversity, the receiver has to collect signal energy from several multi-paths. For this purpose, a Rake receiver allocates so-called fingers to multi-paths. Each finger despreads the receive signal synchronized to the corresponding path delay. The Rake receiver subsequently computes a weighted sum of the Rake fingers' output.

It is important to note, however, that the Rake receiver does not take into account IPI caused by correlation of delayed versions of the spreading codes. Paths separated by less than one chip duration significantly degrade Rake receiver performance.

Multi-path interference caused by paths separated by less than one chip duration is called Inter-Chip Interference (ICI). The despreader does not suppress this interference, because

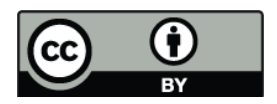

Correspondence to: C. Buchacher (clemens.buchacher@infineon.com) the corresponding signals' pulse shapes overlap. The multipath delay profiles used in High Speed Downlink Packet Access (HSDPA) test scenarios - HSDPA is a high data rate extension of the Universal Mobile Telecommunications System (UMTS) standard - exhibit ICI. In order to achieve higher data rates it is necessary to introduce a more sophisticated algorithm which is capable of suppressing multi-path interference. The chip-level LMMSE equalizer, which maximizes the Signal-to-Interference and Noise Ratio (SINR), is such an algorithm.

Typical channel delay spreads are in the range of $0 \mu$ s to $5 \mu \mathrm{s}$. At the UMTS chip rate of $3.84 \mathrm{MHz}$ and an oversampling factor of two, $5 \mu$ s correspond to roughly $n=40$ receive samples. An LMMSE equalizer of this length exceeds the Rake receiver's computational complexity by far. The LMMSE equalizer coefficient computation involves inversion of a channel autocorrelation matrix of size $n \times n$. Approximating the inverse of this matrix has runtime complexity of at least $\mathbb{O}\left(n^{2}\right)$. Thus, the equalizer's complexity increases significantly with increasing channel length. The complexity of the Rake receiver, on the other hand, is independent of the magnitude of the path delays, because the computation of Rake finger weights does not involve inversion of the channel autocorrelation matrix.

Even though in theory the LMMSE equalizer always performs better than the Rake receiver, this is not true in practice, because the length of an equalizer is limited by the available computing power. In the case of a propagation channel with large delay spread a limited-length equalizer will therefore suffer from interference caused by multi-paths exceeding its observation window.

As a compromise to this steep performance versus complexity tradeoff we will propose a hybrid algorithm. This algorithm will not achieve the performance of an equalizer of arbitrary length, but due to the shorter equalizer length, computational complexity is comparatively small and it is independent of the channel delay spread. 


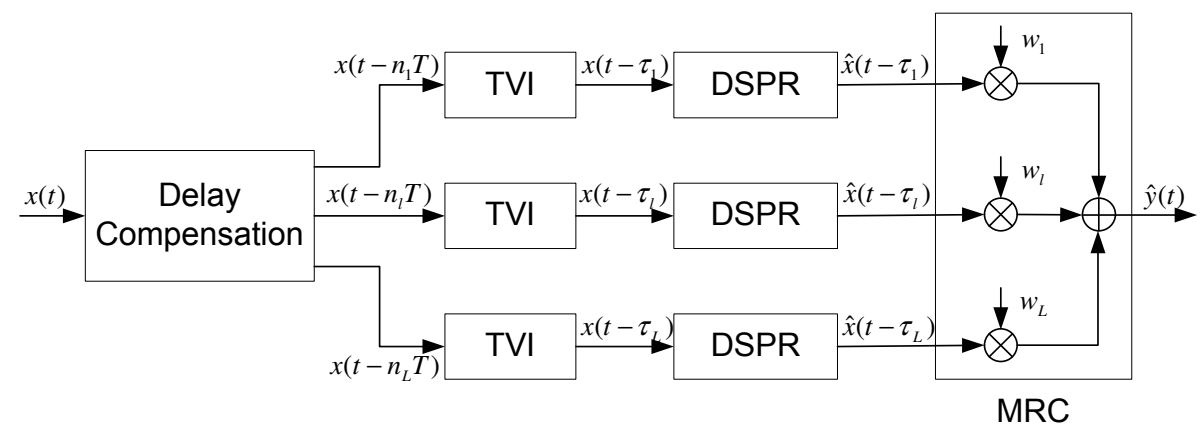

Fig. 1. Symbol-level Rake.

\section{System model}

We consider the CDMA signal

$$
y(t)=\sum_{k \in \mathbb{Z}_{K}} A_{k} \sum_{m \in \mathbb{Z}_{M}} b_{m}^{(k)} \sum_{n \in \mathbb{Z}_{N}} c_{m, n}^{(k)} \delta\left(t-(m N+n) T_{c}\right),
$$

where $\mathbb{Z}_{r}=\{0, \ldots, r-1\}, M$ is the observation block length, $A_{k}$ is the signal amplitude of user $k$ and $\left(b_{0}^{(k)}, \ldots, b_{M-1}^{(k)}\right)$ is the symbol sequence of user $k . N$ is the spreading factor and $\left(c_{m, 0}^{(k)}, \ldots, c_{m,(N-1)}^{(k)}\right)$ is the pseudo-random spreading code sequence for symbol $m$ of user $k . T_{c}$ is the chip duration and $\delta(t)$ is the Dirac delta.

Transmitted over a multi-path channel with additive white Gaussian noise, after matched filtering (matched to the transmit pulse shape) the receiver obtains the signal

$$
\begin{aligned}
x(t) & =y(t) * h(t)+v(t) \\
& =\sum_{\substack{k \in \mathbb{Z}_{K} \\
m \in \mathbb{Z}_{M} \\
n \in \mathbb{Z}_{N}}} A_{k} b_{m}^{(k)} c_{m, n}^{(k)} h\left(t-(m N+n) T_{c}\right)+v(t),
\end{aligned}
$$

where $h(t)$ is the channel impulse response convolved with transmit and receive pulse shaping filters $\psi(t)$ and $\psi^{*}(-t)$, respectively. $\psi(t)$ is non-zero only in the interval $\left[0, T_{c}\right)$. It is sufficient to sample $h(t)$ at twice the chip rate, because we will assume the pulse shape to be band limited to $1 / T_{c}{ }^{1} \cdot v(t)$ is a white Gaussian random process of variance $\sigma^{2}$, filtered by the receive pulse shaping filter.

Without loss of generality the user of interest shall be user number 0 . Furthermore, we will assume the users' power and the channel impulse response to be normalized to unit energy, i.e., $\sum_{k=0}^{K-1} A_{k}^{2}=1$ and $\sum_{l=0}^{L-1}\left|h_{l}\right|^{2}=1$, where $h_{l}=h\left(l T_{c} / 2\right)$ and $L$ is chosen such that $h\left(l T_{c} / 2\right)=0$ for all $l \geq L$.

\footnotetext{
${ }^{1}$ Even though it is impossible for a waveform to be confined to one chip interval and band limited at the same time, the pulse shapes used in Wideband CDMA communications systems approximate these properties reasonably well.
}

\section{Rake receiver}

The Rake Receiver was named after the ordinary garden rake because it collects the different paths by setting a (rake) finger at each corresponding delay. Each finger represents a Time-Variant Interpolator (TVI) used for finesynchronization of the path delay followed by a despreader. Figure 1 depicts the architecture of a Symbol-Level Rake.

For each Rake finger $j$ with the corresponding path delay $\tau_{j}$, the time-variant interpolator eliminates the delay and the despreader reverses the spreading operation. This yields a processing gain equal to the spreading factor $N$. Neglecting multi-path interference, the output Signal-to-Interference and Noise Ratio (SINR) of Rake finger $j$ is

$$
N A_{0}^{2} \frac{\left|h\left(\tau_{j}\right)\right|^{2}}{\rho_{j}+\sigma^{2}},
$$

where

$$
\rho_{j}:=\sum_{\substack{i \in \mathbb{Z} \\ i \neq 0}} \phi_{h}\left(\tau_{j}-i T_{c}\right)
$$

is the cross-correlation induced by other paths and

$$
\phi_{h}(t):=\left|\sum_{l=0}^{L-1} h\left(t-l T_{c} / 2\right)\right|^{2}
$$

is the power delay profile.

The Rake fingers' output is now combined in order to maximize the Signal-to-Noise ratio (SNR). Provided the radio channel adds stationary noise to the receive signal, the output Signal-to-Noise Ratio (SNR) of each finger is proportional to the corresponding path's power. Including phase compensation in the combining operation, the combining weights thus amount to the complex conjugate of the path phasors. This combining scheme is known as Maximum Ratio Combining (MRC).

If we disregard multi-path interference, the output Signalto-Noise Ratio (SNR) after MRC evaluates to

$$
\mathrm{SNR}=N\|\boldsymbol{h}\|_{2}^{2} A_{0}^{2} / \sigma^{2}=N A_{0}^{2} / \sigma^{2},
$$


where $\boldsymbol{h}=\left(h_{0}, \ldots, h_{L-1}\right)^{T}$. Note, however, that this measure can be used as an indicator for the signal quality only if the noise power is dominant over multi-path interference, because MRC is sub-optimum with respect to maximizing the SINR, cf. Burke (2005).

\section{LMMSE equalizer}

Figure 2 shows a block diagram of the discrete random vector model of a linear equalizer. $\boldsymbol{y}, \boldsymbol{n}$ and $\mathbf{H}=\left[h\left((j-i) T_{c} / 2\right)\right]_{i, j}$ denote the discrete time equivalents of the transmit signal, noise signal and channel impulse reponse, respectively. $\boldsymbol{x}$ denotes the random vector obtained by sampling $x(-t)$ at twice the chip rate ${ }^{2}$. $\mathbf{R}$ and $\boldsymbol{r}$ denote the autocorrelation matrix of $\boldsymbol{x}$ and the cross-correlation vector of $\boldsymbol{x}$ with a transmit chip $d$, respectively. In terms of the channel convolution matrix $\mathbf{H}$, noise autocorrelation matrix $\sigma^{2} \hat{\mathbf{I}}$ and equalizer filter delay $R$, they can be expressed as

$$
\begin{aligned}
\mathbf{R} & =E\left(\boldsymbol{x} \boldsymbol{x}^{H}\right)=\mathbf{H} \mathbf{H}^{H}+\sigma^{2} \hat{\mathbf{I}} \text { and } \\
\boldsymbol{r} & =E(\boldsymbol{x} d)=\mathbf{H} \boldsymbol{e}_{R},
\end{aligned}
$$

where $\boldsymbol{e}_{R}$ denotes the $R$-th unit vector.

Given the observation vector $\boldsymbol{x}$ one desires an estimate $\hat{d}$ of the transmit chip $d$ which minimizes the mean square error

$$
J_{\hat{d}}=E\left(|d-\hat{d}|^{2}\right)
$$

where $E(\cdot)$ denotes the expected value. The estimate is also required to be a linear combination of the receive samples $\boldsymbol{x}$, that is

$$
\hat{d}(\boldsymbol{w})=\boldsymbol{w}^{H} \boldsymbol{x},
$$

where the weight vector $\boldsymbol{w}$ is to be determined such that $J_{\hat{d}}$ is minimum. In other words:

$$
\boldsymbol{w}_{\mathrm{o}}=\underset{\mathbf{w}}{\operatorname{argmin}} J_{\hat{d}}(\boldsymbol{w})
$$

This estimator is known as the LMMSE estimator (or equalizer). The solution to this minimization problem is obtained by solving the Wiener-Hopf equation

$$
\mathbf{R} w_{\mathrm{o}}=\boldsymbol{r}
$$

If the noise power is greater than zero $\mathbf{R}$ is guaranteed to be non-singular.

Furthermore, $\mathbf{R}$ is Hermitian and block Toeplitz. These properties are exploited by efficient matrix inversion algorithms such as the conjugate gradient method or the Levinson-Durbin algorithm. Nevertheless, approximating the inverse of an $n \times n$ matrix has runtime complexity of at least $\mathbb{O}\left(n^{2}\right)$. The equalizer length is therefore limited by the available computing power.

\footnotetext{
${ }^{2}$ The discrete time signal vectors are reversed in time to simplify notation of the channel convolution matrix $\mathbf{H}$.
}

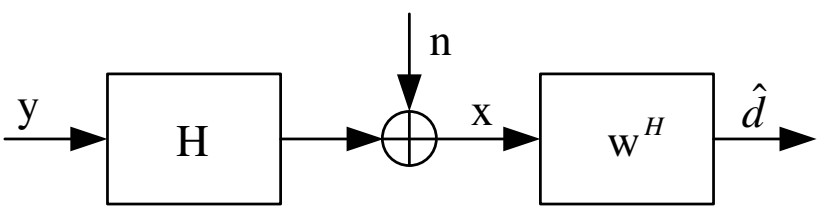

Fig. 2. Discrete random vector model of channel and equalizer.

Provided the observation window is large enough, the equalizer will perform better than the Rake receiver, because in contrast to the Rake receiver the equalizer does not only collect signal energy from the individual paths, but it also reduces multi-path interference. As the channel delay spread gets larger, exceeding the equalizer length, however, the equalizer performance will gradually deteriorate.

\section{Hybrid solution}

Since both of the solutions described in the previous sections are inadequate for high data rate services in multi-path propagation environments with large delay spread, we propose a hybrid approach. Usually, the paths at the beginning of the channel impulse response bear most of the total receive signal energy, because the path power typically decreases exponentially with increasing delay. The channel's strongest source of signal energy and interference can be equalized by a short LMMSE equalizer. The remaining paths not covered by the equalizer are assigned a conventional Rake finger each. This is illustrated in Fig. 3.

In order to motivate this approach we consider the unbiased LMMSE solution. Given an arbitrary transmit vector $\boldsymbol{y}$ and the desired response $d=\boldsymbol{e}_{R}^{T} \boldsymbol{y}$, the estimate's expected value is

$$
\begin{aligned}
E(\hat{d}) & =E\left(\boldsymbol{w}_{\mathrm{o}}^{H} \boldsymbol{x}\right)=\boldsymbol{w}_{\mathrm{o}}^{H} E(\mathbf{H} \boldsymbol{y}+\boldsymbol{n})=\boldsymbol{w}_{\mathrm{o}}^{H} \mathbf{H} e_{R} d \\
& =\boldsymbol{w}_{\mathrm{o}}^{H} \boldsymbol{r} d .
\end{aligned}
$$

The unbiased LMMSE solution is therefore $\boldsymbol{w}_{\mathrm{o}}^{\prime}=\left(\boldsymbol{w}_{\mathrm{o}}^{H} \boldsymbol{r}\right)^{-1} \boldsymbol{w}_{\mathrm{o}}$. In general, the unbiased estimate increases the MSE. The likelihood ratios generated from the symbol estimates for the channel decoder, however, take signal and noise power into account. Introducing an arbitrary scaling factor at this point is therefore of no consequence.

Provided $\sigma^{2} \gg 1$, the signal autocorrelation matrix can be approximated as

$$
\mathbf{R}=\mathbf{H H}^{H}+\sigma^{2} \hat{\mathbf{I}} \approx \sigma^{2} \mathbf{I},
$$

so that the unbiased LMMSE solution reduces to

$$
\boldsymbol{w}_{\mathrm{o}}^{\prime}=\frac{\mathbf{R}^{-1} \boldsymbol{r}}{\boldsymbol{r}^{H} \mathbf{R}^{-1} \boldsymbol{r}} \approx \frac{\boldsymbol{r}}{\boldsymbol{r}^{H} \boldsymbol{r}}=\boldsymbol{r}=\mathbf{H} \boldsymbol{e}_{R} .
$$

As the SNR approaches zero, the unbiased LMMSE equalizer thus degenerates to the Rake receiver. This asymptotic 


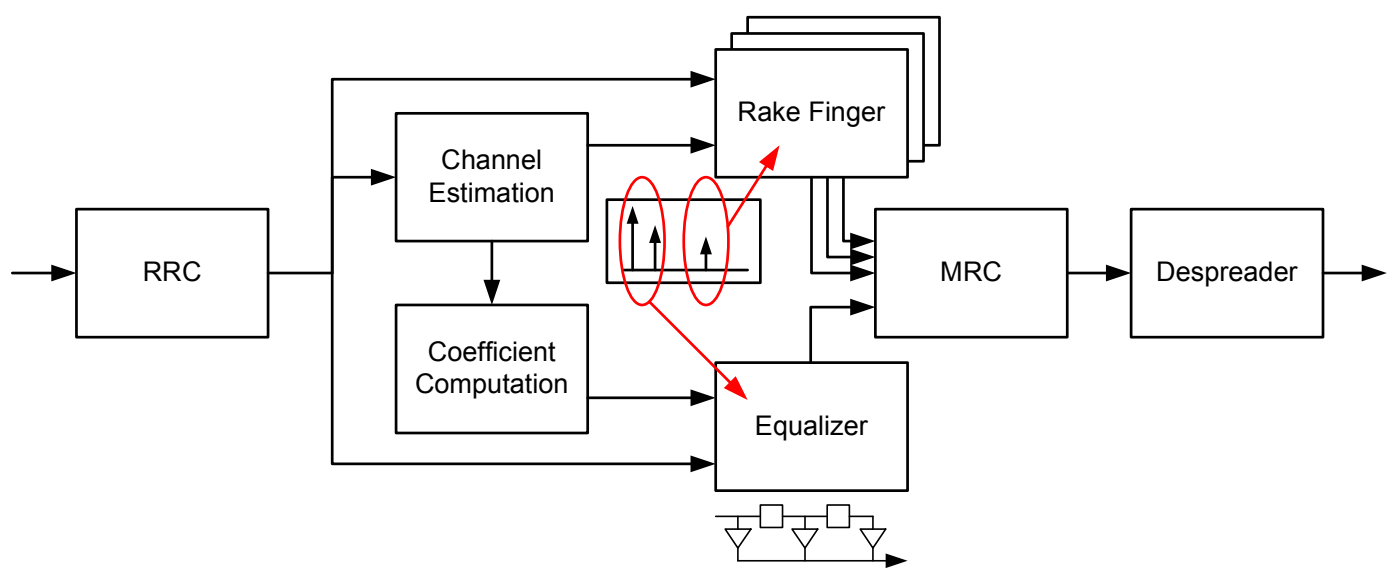

Fig. 3. Combined equalizer/Rake receiver structure.

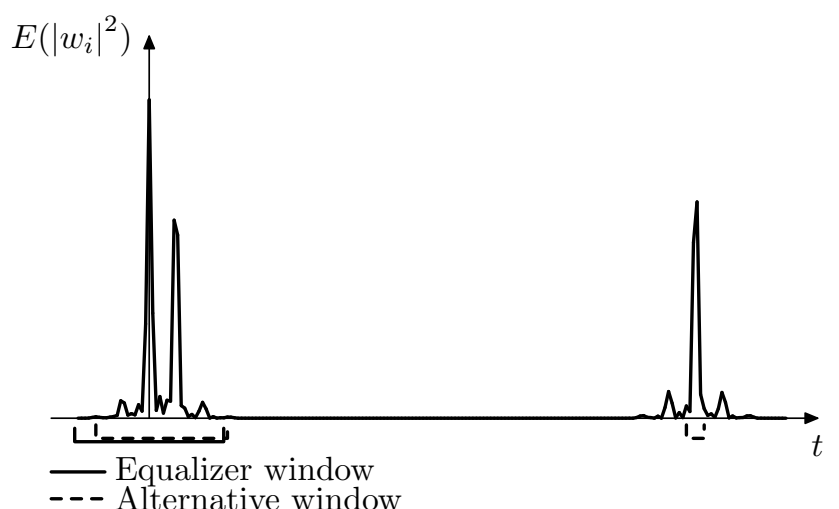

Fig. 4. Case 2 weight statistics for 200-tap equalizer.

behavior can be observed in typical UMTS simulation scenarios as well. Figure 4 shows the average equalizer weight power $E\left(\left|w_{i}\right|^{2}\right)$ simulated for a 200-tap LMMSE equalizer. The weight energy is concentrated around strong path energy. Using Rake fingers as approximation of parts of the equalizer solution is therefore a reasonable approach.

In order to perform MRC of equalizer output and Rake fingers, the equalizer gain has to be estimated. The SINR at the filter output is, cf. Haykin (1996),

$$
\operatorname{SINR}=\frac{E\left(|\hat{d}|^{2}\right)}{J_{\hat{d}}}=\frac{E\left(|d|^{2}\right)-J_{\hat{d}}}{J_{\hat{d}}}=\frac{\boldsymbol{w}^{H} \mathbf{R} \boldsymbol{w}}{J_{\hat{d}}(\boldsymbol{w})} .
$$

If $\boldsymbol{w}$ is the optimum weight vector this relation can be simplified to

$$
\mathrm{SINR}=\frac{\boldsymbol{w}_{\mathrm{o}}^{H} \boldsymbol{r}}{1-\boldsymbol{w}_{\mathrm{o}}^{H} \boldsymbol{r}} .
$$

We thus have the means to estimate the equalizer gain. However, while the equalizer also reduces interference, Rake fingers only minimize noise. Using the equalizer output SINR

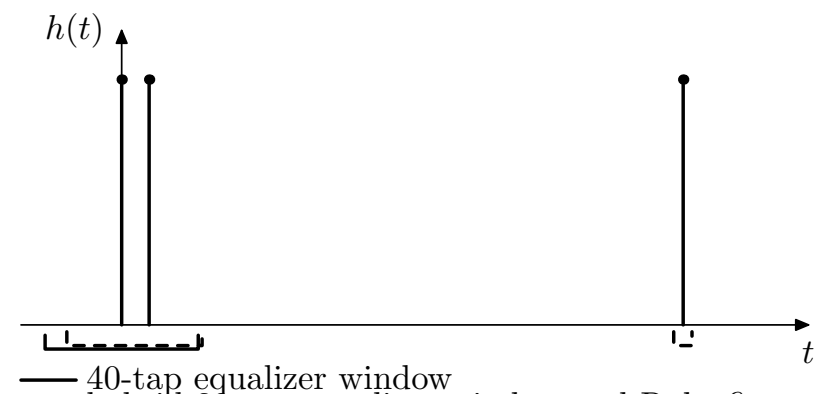

- - hybrid 32-tap equalizer window and Rake fingers

Fig. 5a. Case 2 path delay profile.

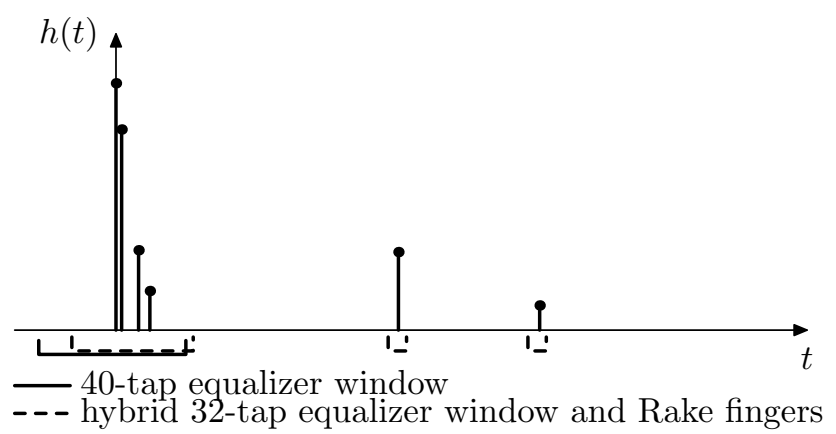

Fig. 5b. PB SP2 $5 \mathrm{~dB}$ path delay profile.

and the SNR of the Rake fingers to estimate the individual receiver path's gain wrongly favors the Rake fingers.

We therefore similarly ignore the interference term, using the output SNR as equalizer gain estimate:

$$
\mathrm{SNR}=\frac{E\left(|\hat{d}|^{2}\right)}{E\left(\left|\boldsymbol{w}_{\mathrm{o}}^{H} \boldsymbol{n}\right|^{2}\right)}-1=\frac{\boldsymbol{w}_{\mathrm{o}}^{H} \boldsymbol{r}}{\left\|\boldsymbol{w}_{\mathrm{o}}\right\|_{2}^{2} \sigma^{2}}-1
$$


Table 1. Case 2 path delay profile.

\begin{tabular}{cc}
\hline Delay $[\mu \mathrm{s}]$ & Attenuation $[\mathrm{dB}]$ \\
\hline 0.0 & 0.0 \\
0.976 & 0.0 \\
20.0 & 0.0 \\
\hline
\end{tabular}

Table 2. PB SP2 $5 \mathrm{~dB}$ path delay profile.

\begin{tabular}{cc}
\hline Delay $[\mu \mathrm{s}]$ & Attenuation $[\mathrm{dB}]$ \\
\hline 0.0 & 0.0 \\
0.2 & 0.9 \\
0.8 & 4.9 \\
1.2 & 8.0 \\
10.0 & 5.0 \\
15.0 & 10.0 \\
\hline
\end{tabular}

\section{Simulation results}

We have implemented an HSDPA simulation chain to simulate performance of the Rake receiver and LMMSE equalizer, as well as the hybrid solution described in the previous section. All simulations use floating-point arithmetic.

The 200-tap equalizer is provided as a reference because it approximates the performance of an infinite-length equalizer. The immense computational complexity of this equalizer makes it infeasible for implementation in a mobile receiver.

The simulation environment is equivalent to the Fixed Reference Channel (FRC) specifications for HSDPA described in 3rd Generation Partnership Project TS 25.101 (2002), which specifies UMTS User Equipment (UE) minimum requirements. Parameter variations are defined for a number of so-called "H-Sets". For example, they specify the number of multi-codes (i.e., the number of parallel HSDPA physical channel codes), which is denoted by $p$ in the performance plots. The parameters $E_{c} / I_{o r}$ and $\hat{I}_{o r} / I_{o c}$ correspond to the desired user's power $A_{0}^{2}$ and the $\mathrm{SNR}=1 / \sigma^{2}$ in our system model, respectively. Performance is measured in terms of HSDPA physical layer throughput. Channel coding and Hybrid Automatic Repeat Request (HARQ) are included in the simulation.

Because delay profiles with large delay spread are of particular interest, instead of the HSDPA minimum requirement test channels, the following power delay profiles are used for performance evaluation.

- The "Case 2" Rake test case, cf. 3rd Generation Partnership Project TS 25.101 (2002), as illustrated in Fig. 5a, is characterized by 3 widely spaced paths of equal power. In particular, this channel exhibits no ICI and

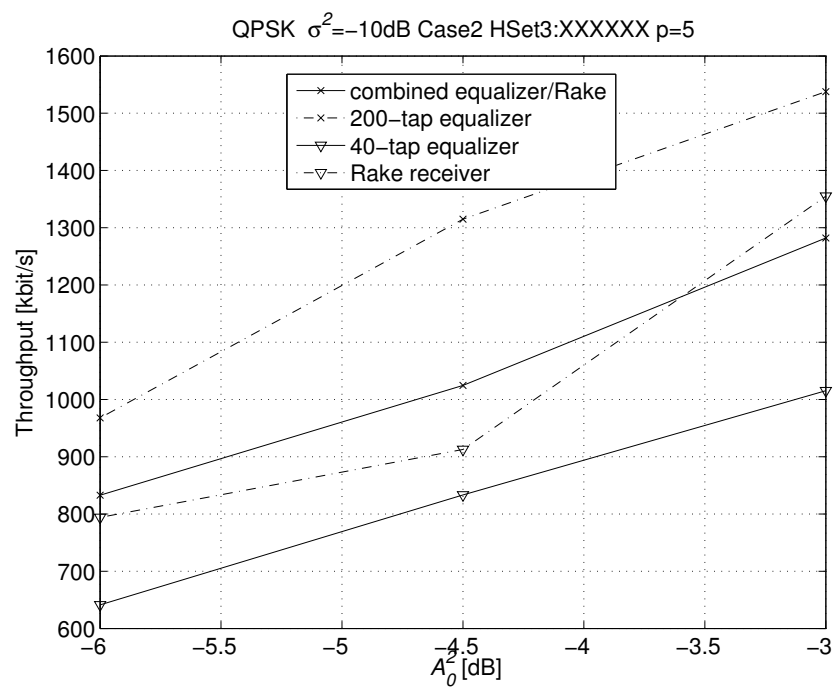

Fig. 6a. Performance results QPSK H-Set $3, \sigma^{2}=-10 \mathrm{~dB}$, Case 2 .

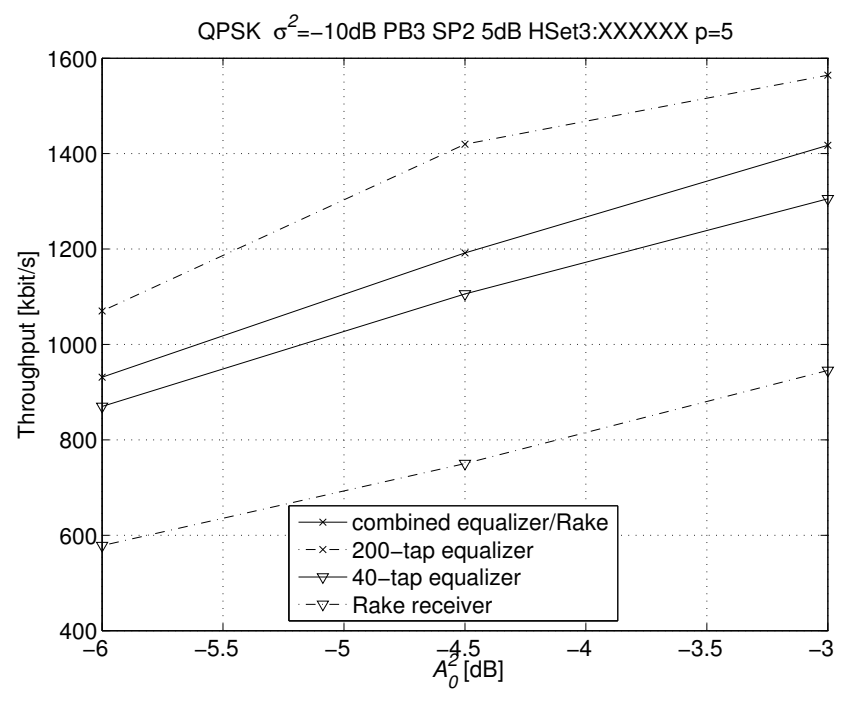

Fig. 6b. Performance results QPSK H-Set $3, \sigma^{2}=-10 \mathrm{~dB}$, PB3 SP $5 \mathrm{~dB}$.

is therefore best suited for the Rake receiver. A conventional equalizer with a manageable number of taps, however, can at best cover the first two taps in its observation window.

- The "PB SP2 $5 \mathrm{~dB}$ " channel is a modified version of the International Telecommunication Union (ITU) Pedestrian B Reference Channel. It introduces two additional paths at $10 \mu$ s and $15 \mu$ s delay, attenuated by $5 \mathrm{~dB}$ and $10 \mathrm{~dB}$, respectively. The delay profile is shown in Fig. 5b. It exhibits both ICI and a large delay spread.

The delay profile specifications are reproduced in Tables 1 and 2 . 


\subsection{Discussion of results}

Performance results for each channel are now discussed individually:

Case 2. Clearly, the Rake receiver is expected to perform better than the 40-tap equalizer for the Case 2 test case. The 40-tap equalizer is not even "aware" of the third path, which simply remains as a significant amount of interference at the equalizer output. Furthermore, the Rake receiver does not have to deal with ICI. Accordingly, Fig. 6a shows that the Rake receiver performance exceeds the equalizer performance by $1.5 \mathrm{~dB}$. The 200 -tap equalizer reference shows that an infinite-length equalizer can still improve performance by approximately $1 \mathrm{~dB}$.

The hybrid solution does not achieve a significant performance gain. The additional Rake finger compensates for the too-short equalizer, however, so its performance still competes with the Rake receiver.

$P B S P 25 d B$. For the modified Pedestrian B channel, the situation is just the opposite. The two late paths are not strong enough to cause too much interference with the 40-tap equalizer. The Rake receiver, however, is impaired by strong ICI, caused by closely spaced paths at the beginning of the delay profile. It comes as no surprise that the plot in Fig. $6 \mathrm{~b}$ shows a huge performance gap between equalizer and Rake receiver. The hybrid solution benefits from the advantages of both the equalizer and the Rake receiver. It achieves a small performance gain over the 40-tap equalizer.

\section{Conclusions}

We have shown that both the Rake receiver and the limitedlength equalizer perform badly in certain scenarios. The Rake receiver performance is severely affected by multi-path interference. An equalizer can reduce multi-path interference, but, for the number of taps which are required to equalize a path with large delay spread, the LMMSE equalizer is computationally too complex.

The solution presented was a hybrid equalizer/Rake receiver. It uses a subset of the available observation window for equalization, allocating Rake fingers to the remaining multi-paths. The equalizer is treated as a single Rake finger, so that after appropriate gain estimation, Rake finger and equalizer outputs can be combined. Our simulation results show that the hybrid algorithm can exceed performance of both, a limited-length LMMSE equalizer and a Rake receiver, with comparative computational complexity in scenarios with strong multi-path interference and large delay spread.

\section{References}

3rd Generation Partnership Project: Technical Specification 25.101: User equipment (UE) radio transmission and reception (FDD), Release 5, 2002.

Burke, J. P., Zeidler, J. R., and Rao, B. D.: CINR difference analysis of optimal combining versus maximal ratio combining, IEEE Transactions on Wireless Communications, 4(1), 15, Jan., 2005, postprint available at: http://repositories.cdlib.org/ postprints/596.

Haykin, S.: Adaptive Filter Theory, Prentice Hall, 3rd Edition, 1996. 